TRANSACTIONS OF THE

AMERICAN MATHEMATICAL SOCIETY

Volume 353, Number 1, Pages 77-93

S 0002-9947(00)02513-7

Article electronically published on June 13, 2000

\title{
INDUCED FORMAL DEFORMATIONS AND THE COHEN-MACAULAY PROPERTY
}

\author{
PHILLIP GRIFFITH
}

\begin{abstract}
The main result states: if $A / B$ is a module finite extension of excellent local normal domains which is unramified in codimension two and if $S / \varkappa S \simeq \hat{B}$ represents a deformation of the completion of $B$, then there is a corresponding $S$-algebra deformation $T / \varkappa T \simeq \hat{A}$ such that the ring homomorphism $S \hookrightarrow T$ represents a deformation of $\hat{B} \hookrightarrow \hat{A}$. The main application is to the ascent of the arithmetic Cohen-Macaulay property for an étale map $f: X \rightarrow Y$ of smooth projective varieties over an algebraically closed field.*
\end{abstract}

The motivation for this article can be described within the following framework. Let $k$ be an algebraically closed field and suppose that $f: X \rightarrow Y$ is a (finite) étale morphism of smooth projective $k$-varieties in which $Y$ is arithmetically CohenMacaulay, that is, there is an ample line bundle $\mathcal{L}$ on $Y$ defining a projective embedding of $Y$ so that the associated homogeneous coordinate ring $\bigoplus_{n} H^{0}\left(Y, \mathcal{L}^{\otimes n}\right)$ is Cohen-Macaulay. In this context we are interested in determining to what extent the Cohen-Macaulay property ascends to $X$ in the sense of: does the line bundle $f^{*} \mathcal{L}$ perform the same feat for $X$ ? For the slightly more special setting in which $Y$ is assumed to be arithmetically Gorenstein, we claimed in [5. Theorem 4.1] that, if $\operatorname{dim} Y \geq 4$, then $X$ necessarily inherits the arithmetic Gorenstein property in the sense stated above. However, it turns out that this result is true only in a rather narrow context. A counterexample was communicated to us by Jonathan Wahl (see Section 4 for a detailed discussion). Wahl's example seemed to suggest that a source of the problem might be found in the behavior of cohomology for fibered products. We found that this was indeed the case. Specifically, in Section 4 we describe a series of examples $f: X \rightarrow Y$ for which a particular embedding of $Y$ determines Cohen-Macaulay homogeneous coordinate rings for $Y$ and $X$. However the induced étale morphism $X \times \mathbb{P}^{e} \rightarrow Y \times \mathbb{P}^{e}, e \geq 1$, with respect to the Segre embeddings determines a Cohen-Macaulay homogeneous coordinate ring for $Y \times \mathbb{P}^{e}$ but fails to do so for $X \times \mathbb{P}^{e}$. While the depth of the homogeneous coordinate ring for the induced Segre embedding of $X \times \mathbb{P}^{e}$ is not sufficient for the Cohen-Macaulay property, nevertheless the depth can grow "large" as the dimensions of $X$ and $\mathbb{P}^{e}$ grow "large".

Received by the editors August 15, 1998.

2000 Mathematics Subject Classification. Primary 13B10, 13B15, 13D10, 13F40; Secondary 13H10, 13N05, 14B07.

Key words and phrases. Cohen-Macaulay local rings, normal domains, ramification, deformations, Segre products.

The author would like to thank the referee for several corrections and helpful suggestions.

* See Added in Proof for correction. 
The somewhat pathological examples discussed in Section 4 are rather general. However, in Sections 1-3 we pursue results on the affirmative side of the matter. The most important of these results and central focus of Sections $1-3$ is Theorem 1.1, a result belonging to the theory of formal deformations of local rings. In order to describe the relation of Theorem 1.1 to the above setting, we return to the étale morphism $f: X \rightarrow Y$, where $\operatorname{dim} Y \geq 2$. Given a projective embedding of $Y$ as described above, one obtains an associated module finite extension $B \hookrightarrow$ $A$ of homogeneous coordinate rings which are finitely generated $k$-algebras. The smoothness of $Y$ and $X$ along with the étale property of $f$ translates into the properties that, away from the irrelevant maximal ideal, the extension $A / B$ is étale (= flat and unramified) and $A$ and $B$ are regular there. When considering the Cohen-Macaulay character of $B$ and $A$ it suffices to consider the "completed" extension $\hat{A} / \hat{B}$, where completion is with respect to the irrelevant maximal ideal in $B$. Within this framework, our main result (Theorem 1.1) states that, if $\operatorname{dim} B \geq 2$ and if $B$ has a formal deformation (see Section 1 for definition) $S$ with respect to a regular sequence $\varkappa$ (i.e., $S / \varkappa S \simeq \hat{B}$ ), then there is a corresponding $S$-algebra deformation $T$ of $\hat{A}$ such that the diagram of ring homomorphisms

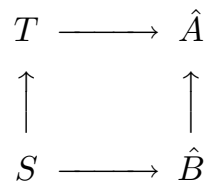

commutes. Restated, Theorem 1.1 says that a formal deformation of $B$ induces a deformation of the ring homomorphism $\hat{B} \rightarrow \hat{A}$. As a consequence (Corollary 1.4) we conclude in case $B$ is Gorenstein that, if the deformation $S$ is "nonsingular" with respect to the punctured spectrum of $\hat{B}$ and if $\operatorname{codim}(\varkappa)>\operatorname{dim} B-4$, then $A$ is Gorenstein also.

In Section 1 we describe several consequences of 1.1. One noteworthy byproduct is a new proof of Cutkosky's purity theorem [7, Theorem 5] for normal complete intersections (see Corollary 1.3). Our proof of Theorem 1.1 is accomplished in Section 2. A key ingredient in our argument makes use of the Hilbert-Burch theorem (see [8, pp. 501-502] for an account of this result). In Section 3 we pursue the existence of "nonsingular generic" formal deformations. The main results (Theorem 3.4 and Corollary 3.5) that emerge here provide sufficient conditions on the conormal module for $B$ (where $B \hookrightarrow A$ is as above) so that the Gorenstein assumption on $B$ ascends to $A$.

For pertinent references in regard to basic notations and unexplained terms, we suggest EGA IV [16, Section 17] with respect to properties of morphisms (unramified, étale, etc.), the books of Eisenbud [8, especially Chapter 16] and Matsumura 23. and Herzog's fundamental paper 19] for deformation theory in commutative algebra, and, finally, the landmark paper of Hochster and Roberts [21. Section 14] for invariant theory and its relation to scheme cohomology for Segre products.

\section{INDUCED FORMAL DEFORMATIONS}

Throughout this section $B$ denotes an excellent, local normal domain. Often $B$ will be Cohen-Macaulay (or even Gorenstein) for applications; however we impose the least number of restrictions on $B$ that are required since some applications call for less (Theorem 1.7). The notation $A / B$ denotes a module finite extension of 
normal local domains. In case $A / B$ is unramified in codimension two our main result (Theorem 1.1) of this article describes a natural correspondence between the formal deformations of $B$ with those of $A$. Thus the formal deformation theory of $A$ (abundance, codimension etc.) is heavily influenced by that of $B$.

By a formal deformation $S$ of a local ring $R$ with respect to an $S$-sequence $\varkappa$, we mean there is a regular sequence $\varkappa$ on a complete local ring $S$ such that $S / \varkappa S \simeq \hat{R}$, where $\hat{R}$ denotes the completion of $R$ with respect to its maximal ideal. The codimension of such a formal deformation is defined to be the codimension of the ideal $\varkappa$ in the local ring $S$.

Theorem 1.1 (Notation as above). If $A / B$ is unramified in codimension two, then any formal deformation $S$ of $B$ with respect to a sequence $\varkappa$ induces a corresponding $S$-algebra formal deformation $T$ of $A$ with respect to $\varkappa$ such that the diagram of ring homomorphisms

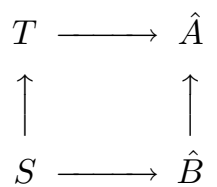

commutes. Moreover, the extension T/S of normal local domains is also unramified in codimension two. (See Added in Proof.)

Remarks 1.2 (Notation as in 1.1). a) One needs the excellence of $B$ in order to ensure that both $\hat{B}$ and $\hat{A}$ are normal domains.

b) If the local ring $B$ satisfies the regularity condition $R_{k}$, then so do $\hat{B}, \hat{A}, S$ and $T$. One needs the purity of branch locus theorem (see [24) as well as "excellence" in order to verify this.

c) If codim $\varkappa=\ell$ and if both $B$ and $S$ are isolated singularities, then $A$ satisfying $S_{j}$, for $j \leq \operatorname{dim} A$, implies that $T$ satisfies $S_{j+\ell}$. That is, in this case the "Serre condition" must grow from $A$ to $T$ by codimension of $\varkappa$.

As an application of our theorem (1.1) on induced deformations we recover Cutkosky's improvement 7, Theorem 5] of Grothendieck's purity of branch locus theorem for normal complete intersections (see [15. Théorème 3.4]).

Corollary 1.3. Let $B$ be an excellent, local normal, complete intersection and suppose that $A / B$ is a module finite extension of local normal domains. If $A / B$ is unramified in codimension two, then $A / B$ is étale.

Proof. After completing both $B$ and $A$ with respect to the maximal ideal of $B$, we may assume that $\hat{A}$ is also a local ring, since $\hat{A}$ will necessarily be a direct product of complete local normal domains. Since $\hat{B}$ is a complete intersection, there is a formal deformation $S$ with respect to a sequence $\varkappa$ such that $S$ is regular local and $S / \varkappa S \simeq \hat{B}$. From Theorem 1.1 we obtain a complete local $S$-algebra $T$ such that $\varkappa$ is a regular sequence on $T, T / \varkappa T \simeq \hat{A}$, and such that $T / S$ is unramified in codimension two. The purity of branch locus theorem for regular rings (see [24]) then gives that $T / S$ is étale, from which it follows that $\hat{A} / \hat{B}$ and $A / B$ are étale.

The next application of Theorem 1.1 concerns the ascent of the Cohen-Macaulay (or in this case the slightly stronger "Gorenstein" property) from $B$ to $A$. It provides an affirmative result along the lines sought after in the flawed result [5], Theorem $5.2]$ which is mentioned in our introduction and discussed in more detail in Section 
4. In particular it speaks directly to the question posed in the introduction as to when the arithmetic Cohen-Macaulay property ascends from $Y$ to $X$ in the context of an étale morphism $f: X \rightarrow Y$ of smooth projective varieties.

Corollary 1.4. Suppose that $B$ is an excellent, local, normal Gorenstein domain of dimension $\geq 3$ such that $B$ is an isolated singularity. We suppose that $A / B$ is a module finite extension of local normal domains which is unramified in codimension one. If $B$ has a formal deformation $S$ with respect to a sequence $\varkappa$ such that $S$ is an isolated singularity also and if $\operatorname{codim} \varkappa>\operatorname{dim} B-4$, then $A$ is necessarily a Gorenstein isolated singularity.

Proof. Let $T$ be the formal deformation of $A$ with respect to $\varkappa$ which is guaranteed by Theorem 1.1. Since $T / S$ is unramified in codimension one, it follows from [12. Corollary 1.2] that the canonical module of $T$ is isomorphic to $T$. From the Hartshorne-Ogus result [18, Theorem 1.6] (see also [10, Corollary 2.7]) it follows that $T$ (and hence also $A$ ) is Gorenstein if $T$ satisfies the Serre condition $S_{j}$ for $j>1 / 2 \operatorname{dim} T$. An application of $1.2(\mathrm{c})$ above yields that $T$ satisfies $S_{2+\ell}$, where $\ell=\operatorname{codim} \varkappa$. Moreover, $2+\ell>1 / 2(\operatorname{dim} B+\ell)=1 / 2 \operatorname{dim} T$ is equivalent to $\ell>\operatorname{dim} B-4$.

Remark 1.5 (Notation as in 1.4). Under the additional assumption that $B$ contains an infinite field one can reach a similar conclusion as in Corollary 1.4 from the requirement that $\operatorname{codim}(\varkappa) \geq e(A)$, where $e(A)$ represents the multiplicity of $A$. Following Huneke's argument [22] and Matsumura's discussion [23, pp. 107-114], one has that

$$
\begin{aligned}
e(A) & =[A: B] e(B)=[A: B] \ell\left(B /\left(y_{1}, \ldots, y_{d}\right) B\right) \\
& =[\hat{A}: \hat{B}] \ell\left(\hat{B} /\left(y_{1}, \ldots, y_{d}\right) \hat{B}\right) \\
& =[\hat{A}: \hat{B}] e(\hat{B}) \\
& =e(\hat{A})
\end{aligned}
$$

where $y_{1}, \ldots, y_{d}$ generates a reduction ideal for the maximal ideal of $B$ and where " $\ell(\bullet) "$ indicates length. As noted in Remark 1.2(c), each lift of $\hat{A}$ as an isolated singularity increases its "Serre depth" $\left(S_{n}\right)$ by one while the multiplicity $e(\hat{A})$ remains constant. However, as soon as the " $n$ " in $S_{n}$ reaches $e(\hat{A})$, then Huneke [22] has observed that $\hat{A}$ (and hence also $A$ ) is necessarily Cohen- Macaulay.

Our example [5, Example 5.5] shows that there are excellent complete intersections $B$ and extensions $A / B$ which are unramified in codimension one for which there can be no deformation of $A$ corresponding to a (regular) deformation of $B$. However, we provide a simpler example to illustrate that the assumption " $A / B$ is unramified in codimension two" cannot be lowered by one (in general) in the statement of Theorem 1.1.

Example 1.6. Let $A=\mathbb{C}[[X, Y]]$ and let $B=\mathbb{C}\left[\left[X^{2}, X Y, Y^{2}\right]\right]$. Then $B$ is the ring of invariants in $A$ under the linear $\mathbb{Z}_{2}$-action defined by $\sigma(X)=-X, \sigma(Y)=-Y$, where $\mathbb{Z}_{2}=\langle\sigma\rangle$. From [9, p. 85] we have that $A / B$ is unramified in codimension one. We note that $A / B$ is not unramified in codimension two, since the maximal ideal of $A$ is clearly ramified. Since $B$ is naturally isomorphic to a complete hypersurface, there is a deformation $S$ of $B$ such that $S$ is regular and $S / x S \simeq B$. However, there cannot be a corresponding deformation $T$ of $A$ as in 1.1. For if there were, the fact 
that $T / S$ would be unramified in codimension one would yield that $T / S$ is étale and, consequently, that $A / B$ is étale (= unramified in dimension two). Thus, one cannot simply replace "codimension two" by "codimension one" in the statement of Theorem 1.1.

Our final applications concern the lifting of codimension one reflexive ideals whose divisor classes represent torsion elements in the divisor class group. This result represents a partial converse to the author's and Weston's result 14, Theorem $1.2]$.

Theorem 1.7. Let $B$ be a complete local normal domain and let $I$ be a reflexive ideal (i.e., $I \simeq \operatorname{Hom}_{B}\left(\operatorname{Hom}_{B}(I, B), B\right)$ ) that is locally principal in codimension two. In addition, we suppose that the class of $I$ in $\mathrm{Cl} B$ has finite order $e$ and that $e$ is relatively prime to the characteristic of the residue field of $B$. If $B$ has a deformation $S$ with respect to the $S$-sequence $\varkappa$ such that $S / \varkappa S \simeq B$, then $I$ lifts to $S$.

Proof. Let $A=B \oplus I z \oplus I^{(2)} z^{2} \oplus \cdots \oplus I^{(e-1)} z^{e-1}$ be the truncation of the "symbolic" Rees algebra described in [4, Section 3] (or see [14, p. 475]). The algebra $A$ is a complete local normal domain and the ring extension $A / B$ is generically Galois with Galois group $\mathbb{Z}_{e}$. Moreover, $A / B$ is unramified in codimension one (see Raynaud [25, pp. 67,68$]$ ); hence $A / B$ is unramified in codimension two since $A$ is $B$-free in codimension two.

From Theorem 1.1 there is an $S$-algebra deformation $T$ of $A$ with respect to $\varkappa$ such that $T$ is a module finite $S$-algebra. It suffices to consider the case $\varkappa=(x)$, since iterating this case will complete the proof. We let $T_{1} / S$ denote the "normal closure" of $T / S$, that is, the associated extension of fraction fields represents the Galois closure for the field extension induced by $T / S$. Let $G=\operatorname{gal}\left(T_{1} / S\right)$, and let $H<G$ be such that $T_{1}^{H}=T$. Since $T_{1}$ is generated by the conjugates of $T$ with respect to $G$, it follows by [1 Proposition A.1] that $T_{1} / S$ is unramified in codimension two. Hence $\bar{T}_{1} / B$ is unramified in codimension one, where $B=S / x S$ and $\bar{T}_{1}=T_{1} / x T_{1}$. This yields that the total quotient ring of $\bar{T}_{1}$ is a product of separable field extensions of the fraction field of $T$. By [14, Lemma 1.1] the integral closure of $\bar{T}_{1}$ is isomorphic to its $B$-double dual $\bar{T}_{1}^{* *}$. Moreover, the $G$ action on $\bar{T}_{1}$ induces, in a unique way, a $G$-action on its integral closure $\bar{T}_{1}^{* *}$ so that $\left(\bar{T}^{* *}\right)^{G}=B$ (note that $|G|=\left[T_{1}: S\right]=\left[\bar{T}_{1}: B\right]=\left[\bar{T}_{1}^{* *}: B\right]$ ). In the same vein, $\left(\bar{T}_{1}^{* *}\right)^{H}=A$. Standard techniques in Galois theory reveal that $H \triangleleft G$, since $A / B$ is generically Galois. Thus $T / S$ is necessarily generically Galois with the same cyclic Galois group as $A / B$. From [13, Theorem 2.2] we get that $T$ is a direct sum of reflexive ideals as an $S$-module, and, from the Krull-Schmidt theorem, that the reduction of $T$ modulo $x$ corresponds - up to isomorphism - to the decomposition $A=B \oplus I \oplus \cdots \oplus I^{(e-1)}$.

Corollary 1.8. If $B$ is an excellent local complete intersection which satisfies the regularity condition $R_{2}$, then the divisor class group of $B$ has no torsion element of order $e$, where $e$ is relatively prime to the characteristic of the residue field of $B$.

Proof. By Theorem 1.7 any such class would lift to a regular deformation of $\hat{B}$. However, this would yield that the class is trivial, i.e., that $e=1$. 


\section{Proof of Theorem 1.1}

Our standing hypothesis is that $B$ is an excellent, local normal domain and $A / B$ is a module finite extension of local normal domains which is unramified in codimension two. The normality of $B$ implies that $A_{\mathfrak{p}} / B_{\mathfrak{p}}$ is flat and unramified (= étale), for $\mathfrak{p} \in \operatorname{Spec} B$, whenever $A / B$ is unramified at $\mathfrak{p}$ (see 1, Proposition 4.3]); hence $A_{\mathfrak{p}} / B_{\mathfrak{p}}$ is étale for codim $\mathfrak{p} \leq 2$. Since the induced extension of fraction fields, $K_{A} / K_{B}$, is necessarily separable, the ramification locus of $A / B$ is defined by an ideal $\mathfrak{A}$ in $A$ as described in 9 , p. 84]. In particular, codim $\mathfrak{A} \geq 3$ and $\hat{\mathfrak{A}}$ defines the ramification locus of $\hat{A} / \hat{B}$; so $\hat{A} / \hat{B}$ is unramified in codimension two. It follows that

(2.1). We may assume that $B$ and $A$ are complete local normal domains.

At this point we add the following ingredients. Let $Y=\left(Y_{1}, \ldots, Y_{d}\right)$ be a set of power series variables such that there is a surjection $\Lambda=B[[Y]] \rightarrow A$ with ideal kernel $I$. Next we set $\Sigma=S[[Y]]$ and note that $\Sigma / \varkappa \Sigma=\Lambda$. We let $J$ be the ideal in $\Sigma$ with the property that $\Sigma / J \simeq A$, where $J$ represents the image of the prime $I$ in Spec $\Sigma$ under the map Spec $\Lambda \rightarrow$ Spec $\Sigma$. Finally, we observe that it suffices to carry out the proof when $\varkappa=(x)$, i.e., when codim $\varkappa=1$.

With the above framework set, we remark that our point of view in constructing the deformation $T$ is as follows. We wish to find a lifting of $A$ as a cyclic $\Lambda$-module, $A=\Lambda / I$, to a cyclic $\Sigma$-module $T=\Sigma / K$ such that $0 \rightarrow T \stackrel{x}{\longrightarrow} T \rightarrow A \rightarrow 0$ is exact. This point of view corresponds to the construction of an "embedded deformation" (see [8] pp. 175-176]). The following observation is an important first step in this construction.

(2.2). $A$ is locally a complete intersection in $\Lambda$ at all primes $P \in V(I) \subseteq$ Spec $\Lambda$ for which codim $P \leq \operatorname{codim} I+2$; that is, the prime ideal $I$ is generated by a $\Lambda_{P}$-sequence at all such prime ideals.

In order to justify (2.2) we consider the conormal sequence for Kähler differentials of $\Lambda$ and $A$ computed over $B$ :

$$
I / I^{2} \stackrel{d}{\longrightarrow} A \otimes_{\Lambda} \Omega_{\Lambda / B} \rightarrow \Omega_{A / B} \rightarrow 0 .
$$

For a prime ideal $P \in V(I)$ with codim $P \leq \operatorname{codim} I+2$ one has that $\left(\Omega_{A / B}\right)_{P}=0$ and the map $d$ is monic at $P$ (see $\left[8,16.12\right.$ (p. 396)]). Since $\Omega_{\Lambda / B}$ is free as a $\Lambda$ module, it follows that $I_{P} / I_{P}^{2}$ is a free $A_{P}$-module of rank $=\operatorname{codim} I$. Furthermore, the ring $\Lambda_{P}=B[[Y]]_{P}$ is Cohen-Macaulay since $B_{P \cap B}$ is Cohen-Macaulay (of dimension 2) and since $B \rightarrow \Lambda$ has regular fibers (see [23. Theorem 23.9, p. 184]). Hence lifting a basis from $I_{P} / I_{P}^{2}$ to $I_{P}$ gives that $I_{P}$ is generated by a $\Lambda_{P}$-sequence. Another consequence of this argument is noted in the next statement.

(2.3). The dual module $\operatorname{Hom}_{A}\left(I / I^{2}, A\right)$ is free as an $A$-module.

The fact that $A$ satisfies the Serre condition $S_{2}$ and that the $A$-modules Ker $d$ and $\Omega_{A / B}$ have no support in codim $\leq 2$ in Spec $A$ and the exact sequence (as above)

$$
0 \rightarrow \operatorname{Ker} d \rightarrow I / I^{2} \rightarrow A \otimes_{\Lambda} \Omega_{\Lambda / B} \rightarrow \Omega_{A / B} \rightarrow 0,
$$

yield an $A$-isomorphism $\operatorname{Hom}_{A}\left(A \otimes_{\Lambda} \Omega_{\Lambda / B}, A\right) \stackrel{\sim}{\longrightarrow} \operatorname{Hom}_{A}\left(I / I^{2}, A\right)$. This proves (2.3). 
In describing the lifting process we follow the paper of Auslander-Ding-Solberg [2, Section 1]. Especially, we make use of the criteria for infinitesimal lifting as set forth in [2, Proposition 1.5]. For this purpose we set $A=A_{1}$ and $J=J_{1}$, where $\Sigma / J_{1}=A_{1}$. We assume that we have in hand an infinitesimal lift $A_{n}$ of $A_{1}, A_{n}=\Sigma / J_{n}$, with respect to the $\Sigma$-ideal $\left(x^{n}\right)$. This means that $\Lambda \otimes_{\Sigma} A_{n} \simeq A_{1}$ and that $\operatorname{Tor}_{i}^{\Sigma}\left(\Lambda, A_{n}\right)=0$ for $i>0$. The obstruction to lifting one more step is contained in the two-fold extension over $\Lambda$ obtained by applying the functor $\Lambda \otimes_{\Sigma}$. to the short exact sequence $0 \rightarrow J_{n} \rightarrow \Sigma \rightarrow A_{n} \rightarrow 0$, from which we get the exact sequence

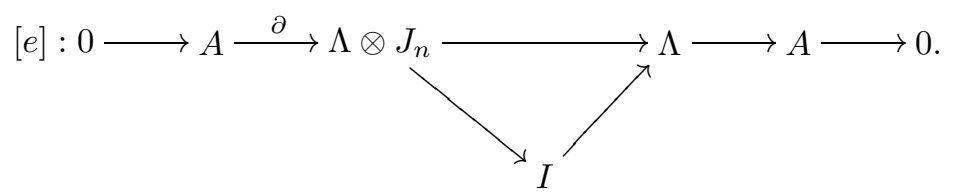

The two-fold extension $[e]$ represents zero in $\operatorname{Ext}_{\Lambda}^{2}(A, A)$ if and only if the monomorphism $\partial$ is split (see discussion in [2] p. 279] following Lemma 1.3). However, we observe that the commutative diagram

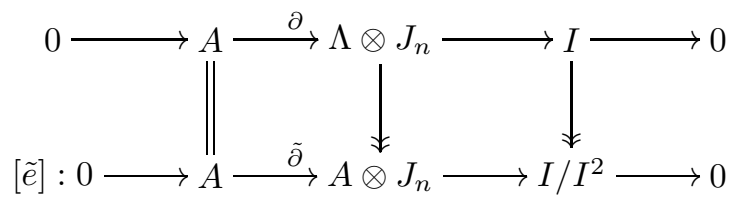

reduces the splitting problem for $\partial$ to the same for $\tilde{\partial}$. It follows that the lifting process can be continued if the bottom short exact sequence represents a split exact extension $[\tilde{e}]$ in $\operatorname{Ext}_{A}^{1}\left(I / I^{2}, A\right)$. Of course the extension representing the bottom short exact sequence is obtained by applying the functor $A \otimes_{\Lambda}$. to the short exact sequence $0 \rightarrow A \stackrel{\partial}{\longrightarrow} \Lambda \otimes J_{n} \rightarrow I \rightarrow 0$. Moreover, (2.2) above ensures that the induced homomorphism $\tilde{\partial}$ is monic, i.e., $J_{n}=\left(x^{n}, y_{1}, \ldots, y_{h}\right)$ and $\tilde{\partial}(1)=x^{n}+I J_{n}$ after localizing at $P \in V(I)$, where codim $P \leq \operatorname{codim} I+2$. (Here we have that $J=\left(x, y_{1}, \ldots, y_{h}\right)$ is a $\Lambda_{P}$-sequence for such $P$.) We record our most recent observations in the following statement.

(2.4). The lifting process may be continued to $A_{n+1}$ provided the extension $[\tilde{e}]$ in $\operatorname{Ext}_{A}^{1}\left(I / I^{2}, A\right)$ represents zero.

In order to verify that (2.4) has an affirmative conclusion, we take a closer look at the short exact sequence $0 \rightarrow A \stackrel{\tilde{\partial}}{\longrightarrow} A \otimes J_{n} \rightarrow I / I^{2} \rightarrow 0$. In particular, we seek more information on the structure of the middle term $A \otimes J_{n} \simeq J_{n} / I J_{n}$. The conormal sequence for the ring surjection $\Sigma \rightarrow A_{n}$ with respect to Kähler differentials computed over $S$ yields the right exact sequence

$$
J_{n} / J_{n}^{2} \rightarrow A_{n} \otimes \Omega_{\Sigma / S} \rightarrow \Omega_{A_{n} / S} \rightarrow 0 .
$$

Combining the relative cotangent sequence [8, 16.2 (p. 386)] for the ring homomorphisms $S \rightarrow B \rightarrow A$ and the conormal sequence for $S \rightarrow A_{n} \rightarrow A$, we obtain the $A$-isomorphisms $\Omega_{A / S} \simeq \Omega_{A / B}$ and $A \otimes \Omega_{A_{n} / S} \simeq \Omega_{A / S}$ (see [8, p. 386]). Thus (*) induces a right exact sequence of $A$-modules

$$
A \otimes J_{n} \rightarrow A \otimes \Omega_{\Sigma / S} \rightarrow \Omega_{A / B} \rightarrow 0 .
$$


We remark that $A \otimes J_{n} \simeq A \otimes J_{n} / J_{n}^{2}$, since $J_{n} \subseteq J_{1}=J$ and $\Sigma / J=A$. In case $Q$ is a prime in Spec $\Sigma$ such that $J \subseteq Q$ and $\operatorname{codim} Q \leq \operatorname{codim} J+2$, one has that $J_{Q}=\left(x, y_{1}, \ldots, y_{d}\right)$ and $\left(J_{n}\right)_{Q}=\left(x^{n}, y_{1}, \ldots, y_{d}\right)$. Here at $Q$ the map $\left(A \otimes J_{n}\right)_{Q} \rightarrow\left(A \otimes \Omega_{\Sigma / S}\right)_{Q}$ becomes an isomorphism, because $\left(\Omega_{A / B}\right)_{Q}=0$ (see the discussion surrounding (2.2)). Since an alternate way of describing this fact is to claim that the $A$-module homomorphism $A \otimes J_{n} \rightarrow A \otimes \Omega_{\Sigma / S}$ is an isomorphism locally over $\operatorname{Spec} A$ in codimension two and since $A$ is a normal domain, we obtain the following useful fact.

(2.5). The $A$-module $\operatorname{Hom}_{A}\left(A \otimes J_{n}, A\right)$ is free.

This claim follows from the preceding discussion together with the observation that $\Omega_{\Sigma / S}$ is a free $\Sigma$-module since $\Sigma=S[[Y]]$.

At this point we are in a position to argue that the obstruction $[\tilde{e}]$ is zero in $\operatorname{Ext}_{A}^{1}\left(I / I^{2}, A\right)$. To this end we consider the $A$-dual exact sequence obtained from $[\tilde{e}]:$

$$
0 \rightarrow\left(I / I^{2}\right)^{*} \rightarrow\left(A \otimes J_{n}\right)^{*} \rightarrow A \rightarrow M \rightarrow 0 .
$$

We are finished provided we can demonstrate that $M=0$, i.e., that $\operatorname{Ass}_{A} M=\emptyset$. Let $P \in \operatorname{Ass}_{A} M$. Then codim $P \geq 3$, since $I / I^{2}$ is $A$-free in codim $\leq 2$. Thus, we obtain a 4 -term exact sequence

$$
0 \rightarrow\left(I / I^{2}\right)_{P}^{*} \rightarrow\left(A \otimes J_{n}\right)_{P}^{*} \rightarrow A_{P} \rightarrow M_{P} \rightarrow 0
$$

in which the first three terms (from the left) are $A_{P}$-free and for which $\ell\left(M_{P}\right)<$ $\infty$ as an $A_{P}$-module. However, this contradicts the structure theorem for free resolutions of length two as described in the Hilbert-Burch theorem (see [8, p. 502]) - or one may view the existence of such a free resolution as being contradictory to the conclusion of the Peskine-Szpiro-Roberts intersection theorem [26, p. 296] (see also [11]). Thus $\operatorname{Ass}_{A} M=\emptyset$ and $M=0$.

Once the above obstructions to lifting are known to vanish, then all that remains is an application of [2] Theorem 1.2] to see that $T=\lim A_{n} \operatorname{lifts} A=A_{1}$ to a $\Sigma$ module $T$ such that $0 \rightarrow T \stackrel{x}{\longrightarrow} T \rightarrow A \rightarrow 0$ is exact. We note that $T=\Sigma / K$, since $A_{1}=A$ is cyclic and since $\Sigma$ is complete in its maximal ideal topology.

Finally we check that the extension $T / S$ is a module finite extension of normal domains that is unramified in codimension two. It is straightforward that $T$ satisfies $R_{1}$ and $S_{2}$; so $T$ is normal. Moreover, $T / S$ is module finite since $T$ is a finitely generated $S$-module modulo $(x), x \in \operatorname{rad} S$, and since $S$ is complete in its (x)-adic topology. In order to check that $T / S$ is unramified in codimension $\leq 2$, we consider the base change of Kähler differentials $A \otimes \Omega_{T / S} \simeq \Omega_{A / S} \simeq \Omega_{A / B}$ that follows from the relative cotangent sequence [8] p. 386]. As a consequence we get the right exact sequence

$$
\Omega_{T / S} \stackrel{x}{\longrightarrow} \Omega_{T / S} \rightarrow \Omega_{A / B} \rightarrow 0
$$

from which one deduces that $\Omega_{T / S}$ vanishes in codimension $\leq 2$ on Spec $T$, since the same statement holds for $\Omega_{A / B}$ on Spec $A$.

(2.6). In regard to the vanishing of the obstruction $[\tilde{e}]: 0 \rightarrow A \rightarrow A \otimes J_{n} \rightarrow$ $I / I^{2} \rightarrow 0$ so that the lifting process may continue (see preceding argument), the single most important feature is the $A$-freeness of the $A$-dual modules $\left(I / I^{2}\right)^{*}$ and $\left(A \otimes J_{n}\right)^{*}$. In case the ring $A$ satisfies the Serre condition $S_{3}$, the weaker condition (stated in (2.3) and (2.4)), that the $A$-homomorphism $I / I^{2} \rightarrow A \otimes \Omega_{A / B}$ is an 
isomorphism in codimension $\leq 2$, would suffice to show $\operatorname{Ext}_{A}^{1}\left(I / I^{2}, A\right)=0$ (see [23. Theorem 16.6]). In the language of [19] Section 1] this vanishing is equivalent to the vanishing of the relative cotangent functor $T^{2}(A / B)$. However, in case $A$ is merely $S_{2}$ the vanishing of $[\tilde{e}]$ is more subtle. Perhaps this subtlety is best seen in the "Grothendieck" context of an étale map of schemes $\left(X_{A}, \mathcal{O}_{A}\right) \rightarrow\left(X_{B}, \mathcal{O}_{B}\right)$, where $X_{A}=\operatorname{Spec} A-\{m\}$ and $X_{B}=\operatorname{Spec} B-\{m\}$. For the sake of simplicity we assume that $X_{B}$ is regular; hence $X_{A}$ will be regular also. For the analogous scheme $\left(X_{\Lambda}, \mathcal{O}_{\Lambda}\right)$ one obtains a short exact sequence $0 \rightarrow \mathcal{I} \rightarrow \mathcal{O}_{\Lambda} \rightarrow i_{*} \mathcal{O}_{A} \rightarrow 0$ of $\mathcal{O}_{\Lambda}$-modules, where $i$ represents the natural inclusion $X_{A} \hookrightarrow X_{\Lambda}$. From above we have that the $\mathcal{O}_{A}$-module $\mathcal{I} / \mathcal{I}^{2}$ is isomorphic to $\mathcal{O}_{A}^{h}$. Moreover, the condition that $A$ is $S_{3}$ translates into the statement

$$
\operatorname{Ext}_{\mathcal{O}_{A}}^{1}\left(\mathcal{I} / \mathcal{I}^{2}, \mathcal{O}_{A}\right) \simeq \operatorname{Ext}_{\mathcal{O}_{A}}^{1}\left(\mathcal{O}_{A}^{h}, \mathcal{O}_{A}\right)=0
$$

However, when $A$ does not satisfy $S_{3}$ one cannot hope that $H^{1}\left(X_{A}, \mathcal{O}_{A}\right) \simeq$ $\operatorname{Ext}_{\mathcal{O}_{A}}^{1}\left(\mathcal{O}_{A}, \mathcal{O}_{A}\right)=0$. In this case, for $\operatorname{dim} A \geq 3$, one obtains the vanishing of $[\tilde{e}]$ by noticing that extensions in $\operatorname{Ext}_{\mathcal{O}_{A}}^{1}\left(\mathcal{O}_{A}^{h}, \mathcal{O}_{A}\right)$ of the form $0 \rightarrow \mathcal{O}_{A} \rightarrow \mathcal{O}_{A}^{h+1} \rightarrow$ $\mathcal{O}_{A}^{h} \rightarrow 0$ split as a result of the Hilbert-Burch theorem [8, p. 502].

\section{Generic Formal Deformations}

Let $k$ be a perfect field and let $(B, m, k)$ denote a local normal domain that is a localization of a finitely generated $k$-algebra. In this section we make some observations - perhaps much of which is folklore - on the existence of formal deformations of $B$. Especially, we are interested in deformations $S$ of $\hat{B}$ such that the regularity condition $R_{i}$ grows by a factor of $\operatorname{codim}(\varkappa)$ from $B$ to $S$, where $\varkappa$ represents the regular $S$-sequence for which $S / \varkappa S \simeq \hat{B}$. According to Corollary 1.4 the existence of such deformations with $\operatorname{codim}(\varkappa)$ sufficiently large plays a major role with respect to the Cohen-Macaulay character of finite type extensions $A$ of $B$ in which $A / B$ is unramified in codimension two. In turn the existence of such finite extensions $A / B$ for which $A$ is not Cohen-Macaulay imposes a restriction on the growth of the regularity condition $R_{i}$ in formal deformations of $B$. The framework in which we describe the "nonsingular" behavior of formal deformations is that of "generic" formal deformations as defined below.

With $(B, m, k)$ as above, we let $\Lambda$ denote a formal power series ring over $k$ such that $\Lambda / I \simeq \hat{B}$, for some ideal $I$. In order to keep the dimension of $\Lambda$ minimal we further assume that $I \subseteq m_{\Lambda}^{2}$, where $m_{\Lambda}$ denotes the maximal ideal of $\Lambda$. By a generic formal deformation $\hat{S}$ of $B$ we mean that, for a set of variables $Y=\left(Y_{1}, \ldots, Y_{h}\right)$ and for $\tilde{\Lambda}$ the completion of $\Lambda[Y]$ at the prime ideal $m_{\Lambda}[Y]$, there are a $\tilde{\Lambda}$-algebra $\widetilde{S}$ and a regular $\widetilde{S}$-sequence $\varkappa$ such that $\widetilde{S} / \varkappa \widetilde{S} \simeq \widetilde{B}$, where $\widetilde{B}$ represents the completion of $B[Y]$ at $m[Y]$.

Our first observation provides a necessary condition for the existence of formal deformations of $B$ in which the Zariski tangent space does not grow.

Proposition 3.1. Let $(B, m, k)$ be as above. If there exists a formal deformation $S$ of $B$ with $S / \varkappa S \simeq \hat{B}$ and $\varkappa \subseteq m_{S}^{2}$, then $I / I^{2}$ must have a free $\hat{B}$-summand of $\operatorname{rank} \geq \operatorname{codim}(\varkappa)$.

Proof. We note that one may replace the local ring $S$ by its completion, i.e., one may assume that $S$ is a complete local ring. This allows one to obtain a commutative 
triangle

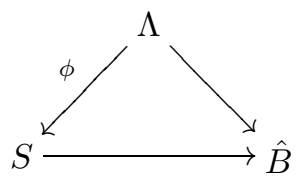

of ring homomorphisms, since $\Lambda$ is a formal power series ring over $k$. Moreover, $m_{S}=\phi\left(m_{\Lambda}\right) S+\varkappa S$ implies $\phi\left(m_{\Lambda}\right) S=m_{S}$ (or, more simply put, $m_{\Lambda} S=m_{S}$ ), since $\varkappa \subseteq m_{S}^{2}$. Thus $\phi(\Lambda)=S$, since $S / m_{\Lambda} S$ is a cyclic $\Lambda$-module and since $S$ is Hausdorff and complete in the $m_{\Lambda}$-topology.

Because each of the ring homomorphisms in the above diagram is a surjection with $\Lambda / I=\hat{B}$ and $S / \varkappa S=\hat{B}$, then $\phi$ induces a $\hat{B}$-surjection $I / I^{2} \rightarrow \varkappa / \varkappa^{2}$. However, the $\hat{B}$-module $\varkappa / \varkappa^{2}$ is necessarily free of rank $=\operatorname{codim}(\varkappa)$.

We keep the notation of 3.1 and observe that the commutative triangle of ring surjections

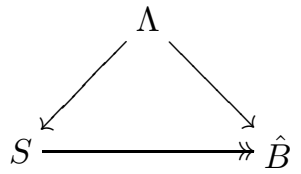

gives $\Lambda / J \simeq S, \Lambda / I \simeq \hat{B}$ and $I=\left(f_{1}, \ldots, f_{d}\right)+J$, where $\left(f_{1}, \ldots, f_{d}\right)$ represents the inverse image of $\varkappa=\left(x_{1}, \ldots, x_{d}\right)$ in $S$. A further analysis of the argument for 3.1 gives the following statement.

Corollary 3.2 (Notation as above). The elements $f_{1}, \ldots, f_{d}$ represent part of a minimal generating set for $I$ at each prime ideal $P$ in $\operatorname{Spec} \Lambda$ which contains $I$.

Proof. At each such $P$ the module $I_{P} / I_{P}^{2}$ maps naturally onto the free $\hat{B}_{P}$-module $\varkappa_{P} / \varkappa_{P}^{2}$, in which the $f_{1}, \ldots, f_{d}$ are sent to a basis of $\varkappa_{P} / \varkappa_{P}^{2}$.

Theorem 3.3. In addition to the standing assumptions on $(B, m, k)$ we assume that $B$ is an isolated singularity, i.e., $\operatorname{Spec} B-\{m\}$ is regular. If $B$ has a formal deformation $S$ such that $S / \varkappa S \simeq \hat{B}$, where $\varkappa \subseteq m_{S}^{2}$, then $B$ has a generic formal deformation $\tilde{S}$ which represents an isolated singularity and for which $\operatorname{dim} \tilde{S}=$ $\operatorname{dim} S$.

Proof. It suffices to consider the case $\varkappa=(x)$, the general case being a finite number of iterations of this case. We use the notation of (3.2) and express $I=(f)+J$, where $\Lambda / I \simeq \hat{B}, \Lambda / J \simeq S$ and where $f$ maps to $x$ in $S$ with $S / x S \simeq \hat{B}$. Let $J=\left(j_{1}, \ldots, j_{h}\right)$ as a $\Lambda$-ideal, and let $Y=\left(Y_{1}, \ldots, Y_{h}\right), Z=\left(Z_{1}, \ldots, Z_{h}\right)$ be two sets of variables. We set $\tilde{\Lambda}=\Lambda[Y, Z]_{m_{\Lambda}[Y, Z]}$ and note that the ideal $\tilde{I}=I \tilde{\Lambda}$ may be expressed as

$$
\tilde{I}=(f)+\tilde{J}
$$

where

$$
\tilde{J}=\left(Y_{1} j_{1}+Z_{1} f, \ldots, Y_{h} j_{h}+Z_{h} f\right) .
$$

In keeping with the above notation, we put $\tilde{S}=\tilde{\Lambda} / \tilde{J}$ and $\tilde{B}=\tilde{\Lambda} / \tilde{I}$ (we bring the respective completions at a later time). 
Let $\mathcal{P} \in \operatorname{Spec} \tilde{S}$. Then $\mathcal{P}$ corresponds to a prime ideal $P \in \operatorname{Spec} \Lambda[Y, Z]$ that contains $\tilde{J}=\left(Y_{1} j_{1}+Z_{1} f_{1}, \ldots, Y_{h} j_{h}+Z_{h} f\right)$. If, in addition, $f \in P$, then $x \in \mathcal{P}$ and there is an exact sequence

$$
0 \rightarrow \tilde{S}_{\mathcal{P}} \stackrel{x}{\longrightarrow} \tilde{S}_{\mathcal{P}} \rightarrow \tilde{B}_{\mathcal{P}} \rightarrow 0 .
$$

It follows that

$$
\tilde{S}_{\mathcal{P}} \text { is regular if } f \in P \text { and } P \neq m_{\Lambda}[Y, Z] .
$$

Also, if $j_{i} \notin P$ for $1 \leq i \leq h$, then

$(* * *) \quad(\Lambda[Y, Z] / \tilde{J})_{P}$ is a localization of $\Lambda\left[j_{1}^{-1}, \ldots, j_{h}^{-1}\right][Y, Z] / \tilde{J}$ which is isomorphic to $\Lambda\left[j_{1}^{-1}, \ldots, j_{h}^{-1}\right]\left[Z_{1}, \ldots, Z_{h}\right]$. Hence $\tilde{S}_{\mathcal{P}}$ is regular in this case as well.

We consider a prime ideal $\mathcal{P} \in$ Spec $\tilde{S}$ such that $\tilde{S}_{\mathcal{P}}$ is not regular when the corresponding $P$ (as above) is properly contained in $m_{\Lambda}[Y, Z]$. Then $j_{i} \notin P$, for $1 \leq i \leq h$, since otherwise $Z_{i} f=\left(Y_{i} j_{i}+Z_{i} f\right)-Y_{i} j_{i} \in P$ implies $f \in P$ and $\tilde{S}_{\mathcal{P}}$ is regular by $(* *)$. However, if $j_{i} \notin P$, for $1 \leq i \leq h$, then $\tilde{S}_{\mathcal{P}}$ is regular from $(* * *)$. Thus, the only prime $P$ such that $\tilde{J} \subseteq P \subseteq m_{\Lambda}[Y, Z]$ and such that $\tilde{S}_{P}$ is not regular is $P=m_{\Lambda}[Y, Z]$. It follows that $\tilde{S}$ is an isolated singularity, and it remains only to complete $\tilde{S}$ and $\tilde{B}$ at the prime ideal $m_{\Lambda}[Y, Z]$ of $\Lambda[Y, X]$.

In the next statement we obtain an affirmative result on the existence of generic formal deformations which satisfy (3.3). This result provides a "generic" converse to (3.1). We call attention to the fact that condition (b) of 3.4 (below) is modeled after a recurring hypothesis used by D. Smith [27.

Theorem 3.4 (Notation as above). We assume that $B$ is an isolated singularity of dimension $\geq 3$ and that the conormal module $I / I^{2}$ for $\widehat{B}$ satisfies:

(a) $I / I^{2}$ has a $\widehat{B}$-free summand of rank $\ell$, and

(b) $\left(I / I^{2}\right)^{*}=\operatorname{Hom}_{B}\left(I / I^{2}, \widehat{B}\right)$ is an $S_{3}$-module.

Then $B$ has a generic formal deformation $\tilde{S}$ with respect to a sequence $\varkappa$ of codimension $\ell$ such that $\tilde{S}$ is an isolated singularity.

Proof. In view of Theorem 3.3 it suffices to demonstrate that $B$ has a formal deformation $S$ such that $S / \varkappa S \simeq \hat{B}$, where $\varkappa$ is a regular sequence on $S$ with $\varkappa \subseteq m_{S}^{2}$. In particular we may neglect the question as to whether or not $S$ is an isolated singularity. Moreover, if we can argue that the essential ingredients for the existence of $S$, where $\varkappa=(x)$ has codimension one, are

(0) $\hat{B}$ satisfies $R_{2}$,

(1) the dual of the conormal module for $\hat{B}$ is $S_{3}$,

(2) the conormal module has a $\hat{B}$-free summand of positive rank,

then we may reduce our argument to the case $\varkappa=(x)$ provided that we demonstrate that $S$ possesses the properties (0), (1), (2) if $\ell>1$.

From [23. Theorem 16.6] we get that $\operatorname{Ext}_{B}^{1}\left(I / I^{2}, \hat{B}\right)=0$. As noted in (2.6) (see also [19] Section 1]) this vanishing is equivalent to the vanishing of $T^{2}(\hat{B} / k)=0$, that is, the vanishing of all obstructions to infinitesimal liftings of $\hat{B}$. Such a $B$ is said to be "unobstructed" (see [19]).

We proceed by choosing $x \in I$ to correspond to a basis element in the free $\hat{B}$-summand of $I / I^{2}$, which exists by hypothesis (a). We write $I=(x)+J$, where

$$
I / I^{2}=\left((x)+I^{2} / I^{2}\right) \oplus\left(J+I^{2} / I^{2}\right) .
$$


We set $I_{1}=I /(x)$, the ideal of relations on $\hat{B}$ in $\bar{\Lambda}=\Lambda /(x)$. From $(*)$ one readily concludes that $I_{1} / I_{1}^{2} \simeq J+I^{2} / I^{2}$. It follows that $\operatorname{Ext}_{B}^{1}\left(I_{1} / I_{1}^{2}, \hat{B}\right)=0$, that is, there are no obstructions to infinitesimal liftings of $B$ from $\bar{\Lambda}$ to $\Lambda$. However, in order to make the technique described in Section $(2.3)$ work one needs that $\left(I_{1}\right)_{P}$ is generated by a $\bar{\Lambda}_{P}$-sequence at each prime ideal in $\bar{\Lambda}$ such that $I_{1} \subseteq P$ and codim $P \leq \operatorname{codim} I_{1}+2$. But this follows from the fact that $x$ is necessarily part of a minimal set of generators for such $P$, since $\hat{B}$ satisfies $R_{2}$. Thus there is a lifting $S$ of $\hat{B}$ from $\bar{\Lambda}$ to $\Lambda$. Hence, $I=(x)+J$, where $J$ is a prime ideal and $x$ is regular on $\Lambda / J=S$. Moreover, the ring $S$ satisfies $R_{2}$, since $B$ satisfies $R_{2}$ and $\operatorname{dim} S=1+\operatorname{dim} B \geq 4$. Therefore part (0) above holds for $S$. In order to verify (1) and (2) above, we begin with the observation that $J \cap I^{2}=x J+J^{2}$ since $J$ is a prime ideal. It follows that there is a right exact sequence

$$
J / J^{2} \stackrel{x}{\longrightarrow} J / J^{2} \rightarrow\left(J+I^{2}\right) / I^{2} \rightarrow 0
$$

where $\left(J+I^{2}\right) / I^{2}$ is a $B$-direct summand of $I / I^{2}$ as stated in $(*)$ above. These facts show that $J / J^{2}$ must contain a free $S$-summand of rank $\ell-1$. Hence (2) above is satisfied. Dualizing the right exact sequence $(* *)$ with respect to $S$, we obtain the left exact sequence

$(* * *) \quad 0 \rightarrow \operatorname{Hom}\left(J / J^{2}, S\right) \stackrel{x}{\longrightarrow} \operatorname{Hom}\left(J / J^{2}, S\right) \stackrel{\rho}{\longrightarrow} \operatorname{Hom}\left(J+I^{2} / I^{2}, B\right)$.

The map $\rho$ is a surjection at all prime ideals in Spec $S$ of codim $\leq 2$, since $J / J^{2}$ is necessarily free at such primes. Therefore, at any prime ideal associated to Cok $\rho$ one has that depth $\operatorname{Hom}\left(J+I / I^{2}, B\right) \geq 3$, since $(J+I) / I^{2}$ is a $B$-direct summand of $I / I^{2}$ and since $\operatorname{Hom}\left(I / I^{2}, B\right)$ satisfies $S_{3}$. A standard local cohomology argument regarding depth implies that $\operatorname{Ass}(\operatorname{Cok} \rho)=\emptyset$, i.e., that $\operatorname{Cok} \rho=0$. So (1) has been verified. Thus (0), (1), (2) hold for $S$. This completes the proof of (3.4).

We conclude this section by combining Corollary 1.4 with Theorem 3.4 in order to achieve an affirmative result on the Cohen-Macaulay property with respect to module finite extensions $A / B$ such that $A / B$ is unramified in codimension two. (Actually $A / B$ will be unramified away from the maximal ideal of $A$, since Spec $B-\{m\}$ is regular.)

Corollary 3.5. We assume that the local normal k-algebra $B$ is a Gorenstein isolated singularity of dimension $\geq 3$. If the conormal module for $\hat{B}$ has a free summand of rank $>\operatorname{dim} B-4$, then any module finite extension $A / B$ of normal domains that is unramified in codim $\leq 1$ must have A Gorenstein as well.

The proof follows from (3.3), (3.4) and (1.4).

\section{4. ÉtALE COVERS AND FIBERED PRODUCTS}

In [5] the author and A. Borek claimed that, if $f: X \rightarrow Y$ is an étale morphism of nonsingular projective varieties over an algebraically closed field $k$ and if $Y$ is arithmetically Gorenstein of dimension $\geq 4$, then so is $X$ arithmetically Gorenstein (see [5, Theorems 3.4 and 4.1]). The important claim here is that the arithmetic Cohen-Macaulay property must ascend from $Y$ to $X$ via the ample line bundle $f^{*} \mathcal{L}$, where $\mathcal{L}$ is an ample line bundle on $Y$ defining an embedding for which the homogeneous coordinate ring is Cohen-Macaulay. Subsequent to the publication of [5], Jonathan Wahl communicated a counterexample to this result. A closer look by the author and Borek at the four-term complex on which their proof rested 
indicated that one of its key properties was not valid (specifically, the conclusion of Lemma 2.2 [5] was not valid in general). Wahl's example was based on an example constructed by A. Beauville [3, Exercise 4, p. 123] in which $f: X \rightarrow$ $Y$ is a finite étale morphism of smooth projective surfaces over $\mathbb{C}$. The variety $X=C \times C$, where $C$ is a plane quintic curve of genus 6 . The first cohomology $H^{1}\left(X, \mathcal{O}_{X}\right)$ has dimension $2 g=12$, which implies that $X$ is not arithmetically Cohen-Macaulay. On the other hand, there is an action of $\mathbb{Z}_{5}$ on $X$ without fixed points such that $Y=X / \mathbb{Z}_{5}$ satisfies " $\rho_{g}=q=0$ " (see [3, Chapter 4]), which gives that the higher cohomology for the structure sheaf on $Y$ vanishes. Although $\operatorname{dim} X=\operatorname{dim} Y=2$, one may form the product cover $X \times X \rightarrow Y \times Y$ and, making use of the Künneth formula for cohomology, achieve a situation in dimension 4 where $Y \times Y$ is arithmetically Cohen-Macaulay while $X \times X$ is not. Of course one may iterate further in order to obtain examples in higher dimensions.

In what follows we demonstrate in a general way that the failure of the arithmetic Cohen-Macaulay property to ascend an étale morphism $f: X \rightarrow Y$ is at least in part a byproduct of the behavior of the cohomology of the fibered product $V \times_{k} W$ in the category of $k$-schemes.

For the sake of simplicity we shall assume throughout this section that $k$ is an algebraically closed field of characteristic zero. The variable behavior of the cohomology of the product $V \times_{k} W$ was first (to our knowledge) studied in a general fashion by Chow [6] in 1964. He wished to determine when the Segre embedding of two projectively Cohen-Macaulay varieties over $k$ was again projectively CohenMacaulay. That is, given projective embeddings $V \hookrightarrow \mathbb{P}_{k}^{r}$ and $W \hookrightarrow \mathbb{P}_{k}^{s}$, with homogeneous coordinate rings $R$ and $S$, respectively, Chow wanted to determine when the homogeneous coordinate ring $R \times_{k} S$ ("Segre product" as defined in [6], [17, pp. 125,126] and [21, p. 1055]) is Cohen-Macaulay. The graded $k$-algebra $R \times{ }_{k} S$ corresponds to the homogeneous coordinate ring of the Segre embedding $V \times W \hookrightarrow \mathbb{P}_{k}^{N}$, where $N=r s+r+s$. As a graded $k$-algebra one has that $\left(R \times{ }_{k} S\right)_{0}=k$ and that $R \times{ }_{k} S$ is generated as a $k$-algebra by all biforms $r \otimes s$ for which $\operatorname{deg} r=\operatorname{deg} s$. As noted by Hochster and Roberts [21, Section 13], the ring $R \times{ }_{k} S$ may be obtained as a ring of invariants of the torus $k^{*}$ acting linearly on $R \otimes_{k} S$ via $g(r)=g^{m} r, g(s)=g^{-m} s$, where $g \in k^{*}$ and where $r \in R, s \in S$ have degree $m$. Thus, if both $R$ and $S$ are normal, it follows that $R \times_{k} S$ is normal.

In his article [6] Chow found sufficient conditions on $R$ and $S$ in order that $R \times_{k} S$ be Cohen-Macaulay (assuming that both $R$ and $S$ are Cohen-Macaulay). We give a brief description of his findings in the spirit of [21, p. 1055]. Let $R$ be a Noetherian graded $k$-algebra and let $x_{1}, \ldots, x_{d}$ be a system of parameters for $R$ that consists of homogeneous elements of the same degree $m$. Then Chow [6] refers to $R$ as proper provided

$$
R_{n} \subseteq\left(x_{1}, \ldots, x_{d}\right) \quad \text { for } \quad n \geq m d .
$$

In case $R$ is Cohen-Macaulay this condition is independent of the system of parameters. Chow [6, p. 818] shows that, if both $R$ and $S$ are Cohen-Macaulay and proper, then $R \times{ }_{k} S$ is Cohen-Macaulay and proper. Moreover, if $R$ and $S$ contain nonzero forms of all positive degrees, then $R \times{ }_{k} S$ Cohen-Macaulay implies that both $R$ and $S$ must be proper. In their famous article [21, Section 14] Hochster and Roberts revisit this issue. Namely, they note that Chow's notion of proper has an interpretation within the cohomology of projective $k$-schemes as follows: Let $R$ be a finitely generated graded $k$-algebra with $R_{0}=k$. We assume that $R$ is 
Cohen-Macaulay and normal. Putting $X=\operatorname{Proj} R$, one has that

$$
R \text { is proper if and only if } H^{r}\left(X, \mathcal{O}_{X}(n)\right)=0
$$

for $n \geq 0$, where $r=\operatorname{dim} X$ (hence $R$ has Krull dimension $r+1$ ).

Having finished with preliminaries, we exhibit a general construction for étale covers $f: X \rightarrow Y$ which demonstrates the variance of the "depth" property for $X$ even though $Y$ is arithmetically Cohen-Macaulay. We let $k[X, Z]:=k\left[X_{1}, \ldots\right.$, $\left.X_{d-1}, Z\right]$ be a polynomial ring over $k$ with $d \geq 5$. We put $f=Z^{d}-\sum_{i=1}^{d-1} X_{i}^{d}$ and set $S:=k[X, Z] /(f)$. The hypersurface ring $S$ represents an isolated singularity as an affine variety over $k$ (i.e., the Jacobian is nonvanishing away from the homogeneous maximal ideal). Noticing that the homogeneous localizations $\left(S_{X_{i}}\right)_{0}$ are regular and recalling that $k$ has characteristic zero, one has that Proj $S$ is a smooth projective $k$-variety. Let $\mathbb{Z}_{d}=\langle\sigma\rangle$ act linearly on $k[X, Z]$ via $\sigma\left(X_{i}\right)=\zeta X_{i}, 1 \leq i \leq d-1$, and $\sigma(Z)=Z$, where $\zeta$ is a primitive $d^{t h}$ root of unity. Since $\sigma(f)=f$, this $\mathbb{Z}_{d^{\text {-action }}}$ on $k[X, Z]$ induces a linear action on $S$ for which the ring of invariants $S^{\sigma}=: R$ has the form (where lower cased letters are used to denote the cosets in $S$ determined by the corresponding variables in $k[X, Z])$

$$
R=k\left[F_{j}\left(x_{1}, \ldots, x_{d-1}\right), z\right]
$$

where the $F_{j}\left(x_{1}, \ldots, x_{d-1}\right)$ vary over all monomials of degree $d$. We list several key properties of the graded $k$-algebras $R$ and $S$ in the next proposition.

Proposition 4.4 (Notation as above). (i) The extension S/R of graded CohenMacaulay normal domains is generically Galois with Galois group $\mathbb{Z}_{d}$.

(ii) The graded k-algebra $R$ is proper in the sense of Chow 6], while $S$ is not.

(iii) The ring extension $S / R$ is étale away from the irrelevant maximal ideal.

(iv) $\operatorname{Proj}(S) \rightarrow \operatorname{Proj}(R)$ represents a finite étale morphism of smooth projective varieties.

Proof. The hypersurface $S$ is of course Cohen-Macaulay and normal. It follows that $R=S^{\sigma}$ is also Cohen-Macaulay and normal (note that $R$ is an $R$-module retract of $S$ since $d=\left|\mathbb{Z}_{d}\right|$ represents a unit in $R$ ). Moreover, $R=S^{\sigma}$, where $\mathbb{Z}_{d}=\langle\sigma\rangle$, implies that the extension of fraction fields is cyclic Galois of order $d$. Hence (i) is established.

In order to see that (ii) holds, we observe that the forms $x_{1}, \ldots, x_{d-1}$ of degree one in $S$ form a homogeneous system of parameters for $S$. However, $z^{d-1} \notin$ $\left(x_{1}, \ldots x_{d-1}\right)$; so $S$ is not proper in the sense of Chow [6] (see 4.1 above). With regard to the properness of $R$ we consider the homogeneous system of parameters $x_{1}^{d}, \ldots, x_{d-1}^{d}$ in $R$. Since $R$ is generated by monomials from $S$ as a $k$-algebra, it suffices to show that each monomial (which lies in $R$ ) of degree $\geq d(d-1)$ is in $\left(x_{1}^{d}, \ldots, x_{d-1}^{d}\right)$. To this end, consider a monomial in $R$ of degree $n \geq d(d-1)$ of the form $z^{i} F$, where $n=i+s$ and where $F=x_{1}^{e_{1}} \cdots x_{d-1}^{e_{d-1}}$ with $s=e_{1}+\cdots+e_{d-1}$. If any $e_{i} \geq d$, then $z^{i} F \in\left(x_{1}^{d}, \ldots, x_{d-1}^{d}\right)$; so suppose that $e_{i}<d$ for $i=1, \ldots, d-1$. This implies that $s<(d-1) d$. However, the fact that $\sigma\left(z^{i} F\right)=\zeta^{s} z^{i} F=z^{i} F \in R=S^{\sigma}$ gives that $d \mid s$. Therefore $s=q d$, where $q<d-1$. Hence $d(d-1) \leq i+s=i+q d$ implies that $i \geq(d-1-q) d>d$. Thus $z^{i} \in\left(x_{1}^{d}, \ldots x_{d-1}^{d}\right)$, since $z^{d}=\sum_{i=1}^{d-1} x_{i}^{d}$. This concludes the argument that $R$ is proper.

To verify that $S / R$ is étale away from the irrelevant maximal ideal, we employ the technique of [9, p. 84] and note that each $x_{i}, 1 \leq i \leq d-1$, is a primitive 
element for the extension of fraction fields. Since $\left(x_{1}, \ldots, x_{d-1}\right)$ generates an ideal primary to the irrelevant maximal ideal, the claim in (iii) follows at once.

From (iii) one sees that $T^{-1} R \rightarrow T^{-1} S$ is étale for any multiplicative set in $R$ which contains a nonzero nonunit element. Hence, for $T=\left\{x_{i}^{d}, x_{i}^{2 d}, \ldots\right\}$ one gets that the extension of zeroth graded pieces $\left(R_{x_{i}^{d}}\right)_{o} \rightarrow\left(S_{x_{i}^{d}}\right)_{o}$ is étale. Since the homogeneous localizations $\left(S_{x_{i}^{d}}\right)_{o}$ are regular, then so are the $\left(R_{x_{i}^{d}}\right)_{o}$. It follows that $\operatorname{Proj}(S) \rightarrow \operatorname{Proj}(R)$ represents an étale morphism of smooth projective varieties over $k$.

The main result of this section follows.

Theorem 4.5 (Notation as above). Let $A=k\left[Y_{0}, \ldots, Y_{e}\right]$ with $e \geq 1$.

(a) The extension $S \times{ }_{k} A / R \times{ }_{k} A$ of finitely generated $k$-algebras represents a finite extension of graded normal domains which is generically Galois with Galois group $\mathbb{Z}_{d}$.

(b) The graded k-algebra $R \times_{k} A$ is Cohen-Macaulay, while $S \times_{k} A$ is not.

(c) $S \times_{k} A$ satisfies the Serre condition $S_{d-1}$, but not $S_{d}$.

(d) The extension $S \times_{k} A / R \times_{k} A$ is étale away from the irrelevant maximal ideal.

(e) $\operatorname{Proj} S \times \mathbb{P}^{e} \rightarrow \operatorname{Proj} R \times \mathbb{P}^{e}$ represents an étale morphism of smooth projective varieties.

Proof. The action of $\mathbb{Z}_{d}=\langle\sigma\rangle$ on $S \times_{k} A$ defined by the formula $\sigma(s \otimes a)=\sigma(s) \otimes a$ on monomials in $S \times{ }_{k} A$ (and then extended by linearity) yields $R \times_{k} A \subseteq\left(S \times_{k} A\right)^{\sigma}$. The normality of $R \times{ }_{k} A$ gives equality and, as a consequence, gives verification of part (a). Part (b) follows from Proposition 4.4 (ii) and the discussion surrounding (4.1).

Part (c) is a consequence of the discussion in the Hochster-Roberts article [21. Remark 14.7, p. 167]. With $W=\operatorname{Proj}\left(S \times_{k} A\right), X=\operatorname{Proj} S$ and $Y=\operatorname{Proj} A$, they note that the Künneth formula gives the isomorphism

$$
H^{\ell}\left(W, \mathcal{O}_{W}\right) \simeq \sum_{n} \bigoplus_{i+j=\ell} H^{i}\left(X, \mathcal{O}_{X}(n)\right) \otimes_{k} H^{j}\left(Y, \mathcal{O}_{Y}(n)\right)
$$

Moreover, for $1 \leq \ell \leq(d-2)+e-1=d+e-3$, the cohomology $H^{\ell}\left(W, \mathcal{O}_{W}(n)\right)$ will vanish except for $\ell=d-2$, where $i=d-2$ and $j=0$, that is, the cohomology $H^{d-2}\left(X, \mathcal{O}_{X}(n)\right) \otimes H^{o}\left(Y, \mathcal{O}_{Y}(n)\right) \neq 0$ for some $n>0$ (see the discussion around (4.2) above). An application of [21, Proposition 5.1, p. 135] implies that the depth of $S \times_{k} A$ at the irrelevant maximal ideal is $d-1$, since $H^{i}\left(W, \mathcal{O}_{W}\right)$ vanishes for $1 \leq i \leq(d-1)-2$, while $H^{d-2}\left(W, \mathcal{O}_{W}\right) \neq 0$. The fact that $S \times_{k} A$ satisfies the Serre condition $S_{d-1}$ will follow from the remainder of the argument, in which it is noted that the local rings of $S \times_{k} A$ are regular (hence Cohen-Macaulay) away from the irrelevant maximal ideal.

Since Proj $S \rightarrow$ Proj $R$ represents an étale morphism of smooth varieties, it follows (see [17] Proposition 10.1 (d)]) that Proj $S \times \operatorname{Proj} A \rightarrow \operatorname{Proj} R \times \operatorname{Proj} A$ is an étale morphism of smooth varieties over $k$. Thus the homogeneous localizations of $S \times_{k} A$ and $R \times_{k} A$ are regular in degree zero. However, the localizations $\left(S \times_{k} A\right)_{x_{i} y_{j}}$ and $\left(R \times_{k} A\right)_{\left(x_{i} y_{j}\right)^{d}}$ will also be regular. Thus (d) and (e) both follow.

In conclusion, one sees, within the context of an étale morphism $X \rightarrow Y$, in which $Y$ is an arithmetically Cohen-Macaulay smooth projective variety over $k$, 
that no amount of depth for $\mathcal{O}_{X}$ (even of the Serre type " $S_{n}$ ") can force $X$ to be arithmetically Cohen-Macaulay. In the end one must rely on additional properties of $Y$ (or $B$, where $\left(Y, \mathcal{O}_{Y}\right)=$ Proj $B$ ), such as those described in Corollary 1.4 or Corollary 3.5 , in order to conclude that $X$ is arithmetically Cohen-Macaulay.

\section{AdDED IN PROOF}

The notation here is consistent with that of Section 2. Recently, Srikanth Iyengar has informed the author that the argument which is presented in "part (**)" of (2.4) in the proof of Theorem 1.1 is not correct as stated. Specifically, the $A$ homomorphism $A \otimes J_{n} \rightarrow A \otimes \Omega_{\Sigma / S}$ is not an isomorphism in codimension $\leq 2$ over Spec $A$ as claimed (in fact the kernel of this homomorphism has rank one). In turn one cannot conclude that $\left(J / J^{2}\right)^{*}$ is free as claimed in $(2.5)$, thus making application of the Hilbert-Burch theorem in (2.5) inappropriate. What the HilbertBurch theorem does provide in (2.5) is that the $A$-dual of the extension $[\tilde{e}]$ is split exact if and only if the $A$-module $\left(J / J^{2}\right)^{*}$ is $A$-free. In case the $\operatorname{ring} A$ satisfies the Serre condition $S_{3}$ this technical point is easily resolved. Namely, the induced homomorphism

$$
\operatorname{Ext}_{A}^{1}\left(\left(I / I^{2}\right)^{* *}, A\right) \rightarrow \operatorname{Ext}_{A}^{1}\left(I / I^{2}, A\right)
$$

is an isomorphism since the kernel and cokernel of the natural homomorphism $I / I^{2} \rightarrow\left(I / I^{2}\right)^{* *}$ have annihilators of codimension $\geq 3$. Since $\left(I / I^{2}\right)^{*}$ is observed to be free in (2.3) it follows that $\operatorname{Ext}_{A}^{1}\left(I / I^{2}, A\right)=0$ and that $[\tilde{e}]: 0 \rightarrow A \stackrel{\tilde{\partial}}{\rightarrow} A \otimes J_{n} \rightarrow$ $I / I^{2} \rightarrow 0$ is split exact. Thus, the lifting process may continue to the next step.

As a result of the above discussion we must add the hypothesis that "the normal domain $A$ is $S_{3}$ " in the statements of Theorem 1.1, Corollary 1.3, Corollary 1.4 and Corollary 3.5. Moreover, the statement in Corollary 1.7 must be amended by stating that "the divisorial ideal $I$ and all of its symbolic powers are $S_{3}$-modules".

Finally the $S_{3}$-assumption needed for the proofs of the above results has the affect of negating the discussion whch takes place in (2.6) since this assumption has the blanket affect of removing all obstructions to lifting $A$ once the base $\operatorname{ring} B$ has a suitable deformation.

\section{REFERENCES}

1. M. Auslander and D. Buchsbaum, On ramification theory in Noetherian rings, Amer. J. Math. 81 (1959), 749-765. MR 21:5659

2. M. Auslander, S. Ding and $\emptyset$. Solberg, Liftings and weak liftings of modules, J. of Alg. 156 (1993), 273-317. MR 94d:16007

3. A. Beauville, Complex Algebraic Surfaces, London Math. Soc. Lecture Notes Series, Vol. 68, Cambridge Univ. Press, Cambridge, 1983. MR 85a:14024

4. J. Bingener and U. Storch, Zur Berechnung der Divisorenklassengruppen kompleter lokaler Ringe, Nova Acta Leopoldina N.F. 52 Nr. 763 (1981), 7-63. MR 83m:13017

5. A. Borek and P. Griffith, Weak purity for Gorenstein rings is determined in codimension four, J. Algebraic Geometry 5 (1996), 415-437. MR 97b:13030

6. W. L. Chow, On unmixedness theorems, Amer. J. Math 86 (1964), 799-822. [MR 30:2031]

7. S. D. Cutkosky, Purity of the branch locus and Lefschetz theorems, Compositio Mathematica 96 (1995), 173-195. MR 96h:13023

8. D. Eisenbud, Commutative Algebra with a view toward Algebraic Geometry, Springer, BerlinHeidelberg-New York, 1994. [MR 97a:13001]

9. R. Fossum, The Divisor Class Group of a Krull Domain, in Ergebnisse der Mathematik und iher Grenzgebiete, vol. 74, Springer, Berlin-Heidelberg-New York, 1973. MR 52:3139 
10. R. Fossum, H. Foxby, P. Griffith and I. Reiten, Minimal injective resolutions with applications to dualizing modules and Gorenstein modules, Inst. Hautes Études Sci. Publ. Math. 45 (1976), 193-215. MR 53:392

11. H. Gillet and C. Soulé, $K$-théorie et nullité des multiplicités d'intersection, C. R. Acad. Sc. Paris Série I Math. 300 (1985), 71-74. MR 86k:13027

12. P. Griffith, Normal extensions of regular local rings, J. of Alg. 106 (1987), 465-475. MR 88c:13020

13. 473-490. MR 92c:13017

14. P. Griffith and D. Weston, Restrictions of torsion divisor classes to hypersurfaces, J. of Alg. 167 (1994), 473-487. MR 95c:13008

15. A. Grothendieck, Cohomologie locale des faisceaux cohérents et théormes de Lefschetz locaux et globaux, Séminaire de Géométrie Algébrique du Bois-Marie, 1962 (SGA2), fasc. 1, Inst. Hautes Études Sci., Paris, 1962; 3rd ed., 1965; reprint, Adv. Stud. Pure Math., vol. 2, NorthHolland, Amsterdam, and Masson, Paris, 1968. MR 35:1604 MR 57:16294

16. L Élements de géométrie algébrique (EGA), Part IV: Étude locale des schémes et des morphismes de schémes. IV, Inst. Hautes Études Sci. Publ. Math. No. 32 (1967). MR 39:220

17. R. Hartshorne, Algebraic Geometry, Springer, Berlin-Heidelberg-New York, 1977. MR 57:3116

18. R. Hartshorne and A. Ogus, On the factoriality of local rings of small embedding codimension, Communications in Algebra 1 (1974), 415-437. MR 50:322

19. J. Herzog, Deformationen von Cohen-Macaulay Algebren, J. Reine Angew. Math. 318 (1980), 83-105. MR 81m:13012

20. M. Hochster and J. Eagon, Cohen-Macaulay rings, invariant theory, and the generic perfection of determinantal loci, Amer. J. Math. 93 (1971), 1020-1058. MR 46:1787

21. M. Hochster and J. Roberts, Rings of invariants of reductive groups acting on regular rings are Cohen-Macaulay, Advances in Math. 13 (1974), 115-175. MR 50:311

22. C. Huneke, A remark concerning multiplicities, Proc. AMS 85 (1982), 331-332. MR 83m:13016

23. H. Matsumura, Commutative Algebra, Cambridge Studies in Advanced Mathematics no. 8, Cambridge Univ. Press, Cambridge, 1989. MR 42:8213; MR 82i:13003 (earlier eds.)

24. M. Nagata, On the purity of branch locus in regular local rings, Ill. Jour. of Math. 3 (1959), 328-333. MR 21:5660

25. M. Raynaud, Anneaux Locaux Henséliens, in Lecture Notes in Mathematics, no. 169, Springer, Berlin- Heidelberg-New York, 1970. MR 43:3252

26. P. Roberts, Multiplicities and Chern Classes in Local Algebra, in Cambridge Tracts in Mathematics, no. 133, Cambridge Univ. Press, Cambridge (1998). CMP 99:13

27. D. Smith, Ph.D. Thesis, University of Illinois (1998).

Department of Mathematics, University of Illinois, 1409 West Green Street, UrBANA, IlLINOIS 61801

E-mail address: griffith@math.uiuc.edu 